Erratum: Spatiotemporal Analysis of Overall Health in the United States Between 2010 and 2018

Binod Acharya ${ }^{1}$, Loni Tabb ${ }^{2}$

1. Urban Health Collaborative, Drexel University, Philadelphia, USA 2. Department of Epidemiology and Biostatistics, Drexel University, Philadelphia, USA

Corresponding author: Binod Acharya, ba525@drexel.edu

Corresponding author: Binod Acharya

1. Urban Health Collaborative, Drexel University, Philadelphia, USA 2. Department of Epidemiology and Biostatistics, Drexel University, Philadelphia, USA

How to cite this erratum

Acharya B, Tabb L (December 17, 2021) Correction: Spatiotemporal Analysis of Overall Health in the United States Between 2010 and 2018. Cureus 13(12): c56. doi:10.7759/cureus.c56

\section{Correction Notice}

This article has been corrected to include Dr. Loni Tabb as second author. Dr. Tabb was erroneously omitted from the article due to a misunderstanding by the first author, Binod Acharya, regarding what constitutes authorship. Both Mr. Acharya and Dr. Tabb have agreed to this formal correction adding Dr. Tabb as second author. Additionally, the acknowledgement of Dr. Tabb has now been removed. 